\title{
Elimination of bistability in constant-phase mode in atomic force microscopy
}

\author{
LI YingZi ${ }^{1,2,3}$, QIAN JianQiang ${ }^{1,3 *}$, LI XiaoFeng $^{2}$, LI Yuan $^{1,3}$, HUA BaoCheng $^{2,3}$ \& \\ YAO JunEn ${ }^{1,2,3}$ \\ ${ }^{1}$ Department of Applied Physics, Beihang University, Beijing 100191, China; \\ ${ }^{2}$ School of Instrumentation Science and Opto-electronics Engineering, Beihang University, Beijing 100191, China; \\ ${ }^{3}$ Laboratory of Micro-nano Measurement, Manipulation and Physics, Ministry of Education, Beihang University, Beijing 100191, China
}

Received April 6, 2011; accepted May 23, 2011

\begin{abstract}
By presenting the phase properties of bistability in amplitude-modulation atomic force microscopy, we put forward a technique, the constant-phase mode, which may eliminate bistability. Using this approach, we keep the phase shift between driving and oscillation constant, slightly above $-90^{\circ}$. In addition to the adjustment of the free amplitude, we add to amplitude-modulation atomic force microscopy another feedback so that the tip always oscillates in the high-amplitude state. A numerical simulation is carried out to demonstrate that the algorithm prevents bistability effectively.
\end{abstract}

bistability, constant-phase mode, atomic force microscopy

Citation: $\quad$ Li Y Z, Qian J Q, Li X F, et al. Elimination of bistability in constant-phase mode in atomic force microscopy. Chin Sci Bull, 2012, 57: 460-465, doi: $10.1007 / \mathrm{s} 11434-011-4825-0$

Atomic force microscopy (AFM) [1] is a physics-based surface analysis using multiple modern technologies. Since its invention in 1986, AFM has rapidly developed into a major high-resolution tool for nanotechnology research $[2,3]$. More advanced techniques have been proposed to improve the performance of AFM [4,5].

Among dynamic imaging modes, amplitude-modulation AFM [6] (AM-AFM) is the simplest and most commonly used; however, it also faces a serious operational challenge of imaging bistability [7-13]. This problem may lead to a false image or instability of a scanned image, compromising the reliability of that image. Because AM-AFM is widely used, how to eliminate the bistability has become an important issue.

It may be possible to reduce the bistability by increasing the free amplitude $A_{0}$, enlarging the cantilever spring constant $k_{\mathrm{c}}$, or selecting an appropriate set amplitude $A_{\text {set }}$ [14]. However, increasing the free amplitude makes it more like-

\footnotetext{
*Corresponding author (email: qianjq@ @uaa.edu.cn)
}

ly to damage the probe and sample, and harder probes reduce the scanning image resolution. In addition, it is always a problem to choose an appropriate set amplitude. It is thus necessary to develop other methods to reduce bistability.

Solares $[15,16]$ proposed the combination of AM-AFM with frequency-modulation AFM to yield frequency and amplitude modulation AFM (FAM-AFM) and frequency and force modulation AFM (FFM-AFM). Although both methods prevent imaging bistability, the experimental implementation of FAM-AFM can be challenging because of the problem of two control loops, where the external loop requires the internal loop to be stable to accurately reproduce the sample topography. The FFM-AFM instrument does not adjust the cantilever rest position during imaging, and sharp topographical features are thus expected to provide the greatest challenges.

Phase shift is one of the main parameters in identifying the transition between low- and high-state in the bistability. In this work, from discussion of the bistability nature, a new algorithm is proposed to eliminate bistability and its feasi- 
bility is analyzed. Its effectiveness in eliminating bistability is demonstrated in a simulation.

\section{Methods and analysis}

\subsection{Equation of motion}

There are many models describing the motion characteristics of the probe in AFM. Two important models are the spring oscillator model and the high-harmonics model based on the improved Euler-Bernoulli equation $[17,18]$. To analyze the effect of the interaction between the tip and sample on the tip's amplitude and phase, the spring oscillator model is sufficiently accurate and is the model we adopt in this work. In this model, the AFM's cantilever is taken as a spring, the probe as a sphere, and the sample surface as a flat surface. The movement of the cantilever is then governed by [19]

$$
m \frac{\mathrm{d}^{2} z}{\mathrm{~d} t^{2}}=-k_{\mathrm{c}} z-\frac{m \omega_{0}}{Q} \frac{\mathrm{d} z}{\mathrm{~d} t}+F_{0} \cos (\omega t)+F\left(z_{\mathrm{c}}, z\right),
$$

where $z$ is the tip-sample distance, $z_{\mathrm{c}}$ the cantilever's equilibrium position, $m$ the cantilever's effective mass, $Q$ the quality factor, $k_{\mathrm{c}}$ the elastic constant, $F_{0}$ the excitation force, $\omega_{0}$ the resonant frequency, and $\omega$ the excitation frequency. In the following calculation, the tip-sample interaction $F\left(z_{c}, z\right)$ is based on the Derjaguin-Mäuller-Toporov model [20]. The interaction is calculated differently according to the interatomic distance $a_{0}$. For distances larger than $a_{0}$, the tip-sample interaction is calculated from the van der Waals force between the sphere and surface [20,21]. For distances smaller than $a_{0}$, the repulsive force between the tip and sample is simulated by an indentation force derived from Hertz's model [21,22]. Furthermore, it is assumed that the viscosity force is proportional to the deformation velocity and contact area. Thus, the interaction force in the contact region is given as [19]

$$
\begin{aligned}
F\left(z_{\mathrm{c}}, z\right)= & -\frac{A R}{6\left(z_{\mathrm{c}}+z\right)^{2}}, z_{\mathrm{c}}+z>a_{0}, \\
F\left(z_{\mathrm{c}}, z\right)= & -\frac{A R}{6 a_{0}^{2}}+\frac{4 E^{*} \sqrt{R}}{3-3 \gamma^{2}}\left(a_{0}-z-z_{\mathrm{c}}\right)^{\frac{3}{2}} \\
& -\frac{\pi \eta R}{h}\left(a_{0}-z-z_{\mathrm{c}}\right) \frac{\mathrm{d} z}{\mathrm{~d} t}, z_{\mathrm{c}}+z \leqslant a_{0},
\end{aligned}
$$

where $A$ is the Hamaker constant, $R$ is the radius of the tip, $E$ and $v$ are respectively the elastic modulus and Poisson ratio, $E^{*}=\left[\left(1-v_{\text {tip }}^{2}\right) / E_{\text {tip }}+\left(1-v_{\text {sample }}^{2}\right) / E_{\text {sample }}\right]^{-1}, \eta$ is the viscosity coefficient, and $h$ is the thickness of the sample.

\subsection{Bistability}

Unless otherwise stated, we assume a tip curvature radius $R=20 \mathrm{~nm}$, cantilever free resonant frequency $f_{0}=44.8 \mathrm{kHz}$ and excitation frequency $f=44.8 \mathrm{kHz}$, free oscillation amplitude $A_{0}=10 \mathrm{~nm}$, cantilever constant $k_{\mathrm{c}}=15 \mathrm{~N} / \mathrm{m}$, quality factor $Q=100$, Hamaker's constant $A=10^{-19} \mathrm{~J}$, sample and probe Poisson coefficients $v_{\text {sample }}=v_{\text {tip }}=0.3$, sample Young modulus $E_{\text {sample }}=1.2 \mathrm{GPa}$, probe modulus $E_{\text {tip }}=130 \mathrm{GPa}$, viscosity coefficient $\eta=0$, and interatomic distance $a_{0}=$ $0.25 \mathrm{~nm}$.

The oscillation amplitude $A$ is the critical experimental parameter measured in AM-AFM. It reflects the change in sample height. The numerical solution of the probe motion equation shows that too large $E^{*}$ or too small $\eta$ may lead to two different solutions, a low-amplitude solution and highamplitude solution, $Z_{\mathrm{H}(\mathrm{L})}=Z_{0}+A_{\mathrm{H}(\mathrm{L})} \cos \left(\omega t-\varphi_{\mathrm{H}(\mathrm{L})}\right)$ [14].

The dependence of the low- and high-oscillation solutions on the remainder of the tip-surface separation is plotted in Figure 1(a). The amplitude curve shows the separation as the tip retracts (solid line) and then approaches from (dotted line) the surface. When the tip-sample distance is 6.9 to $10 \mathrm{~nm}$, both branches provide a value of the amplitude that matches the tip-sample distance; i.e. there is bistability of the solid line for the high state and the dotted line for the low state.

\subsection{Relationship between phase shift and bistability}

The dependence of the phase shift between a tip position signal and excitation signal on the remainder of the tip-surface separation is plotted in Figure 1(b). The parameters are the same as in Figure 1(a). The solid line corresponds to the high state and dotted line to the low state. From $-90^{\circ}$, the phase shift in the low state decreases as the tip-surface separation decreases. When the separation is $6.9 \mathrm{~nm}$, there is a change in the phase shift from the low to the high state; i.e. from below $-90^{\circ}$ to above $-90^{\circ}$. In the high state, different behavior is observed. The phase shift decreases monotonically from $0^{\circ}$ to $-90^{\circ}$ as the tip-surface separation increases. The phase shift is always greater than $-90^{\circ}$ before the tip

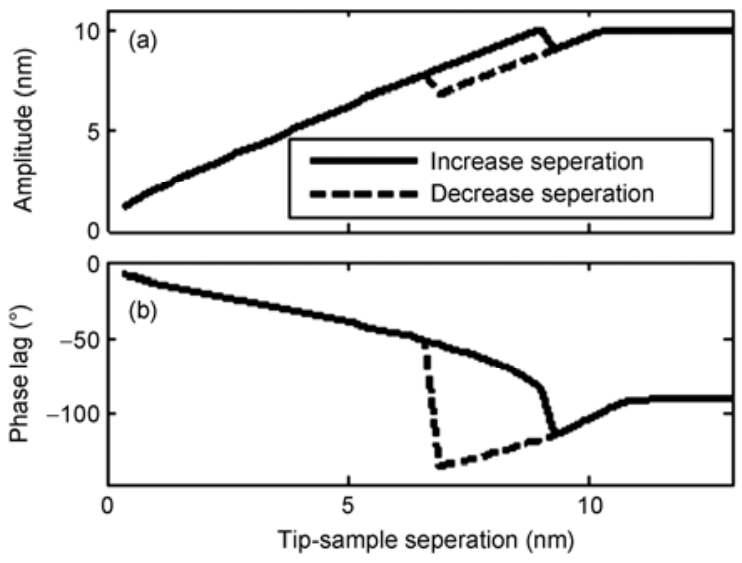

Figure 1 (a) Amplitude as a function of tip-sample separation; (b) phase shift as a function of tip-sample separation. 
amplitude equals the free amplitude.

On the basis of the above, when the tip is working in the high state, the phase shift is maintained between $-90^{\circ}$ and $0^{\circ}$, while the phase shift is maintained between $-180^{\circ}$ and $-90^{\circ}$ in the low state. Experimental phase-shift curves reproducing the above features have been obtained for $\mathrm{SiO}_{2}$, polyvinyl alcohol and mica surfaces [23-26].

Figure 2 presents the scanning images and its phase shift in the amplitude-modulation mode. The dotted line is the sample curve, the solid line the forward curve and the dashed line the backward curve. The parameters of the scanning image are free oscillation amplitude $A_{0}=10 \mathrm{~nm}$, working amplitude set at $A_{\text {set }}=9 \mathrm{~nm}$, scanning speed $v=20$ $\mu \mathrm{m} / \mathrm{s}$, ratio of sampling period to oscillation period of 10 , and proportional-integral-derivative (PID) parameters $k_{\mathrm{P}}=$ $0.04, k_{\mathrm{I}}=0.2$, and $k_{\mathrm{D}}=0.0$.

As in Figure 1, bistability is observed when the amplitude falls between 6.9 and $10 \mathrm{~nm}$. When the amplitude is set at $9 \mathrm{~nm}$, the system is prone to bistable behavior as long as the sample height changes.

The trace and retrace scan lines are presented in Figure 2(a). These two lines do not coincide with each other. The deviation in the vertical height between forward and backward imaging is about $1.85 \mathrm{~nm}$. This deviation is exactly the amplitude difference between the two states. Therefore, the scanning image's instability is caused by the bistability and the system produces a false image.

Figure 2(b) clearly shows the bistability effect on the scanning image. In the forward curve, the phase shift is less than $-90^{\circ}$ and the tip works in the low state initially. When

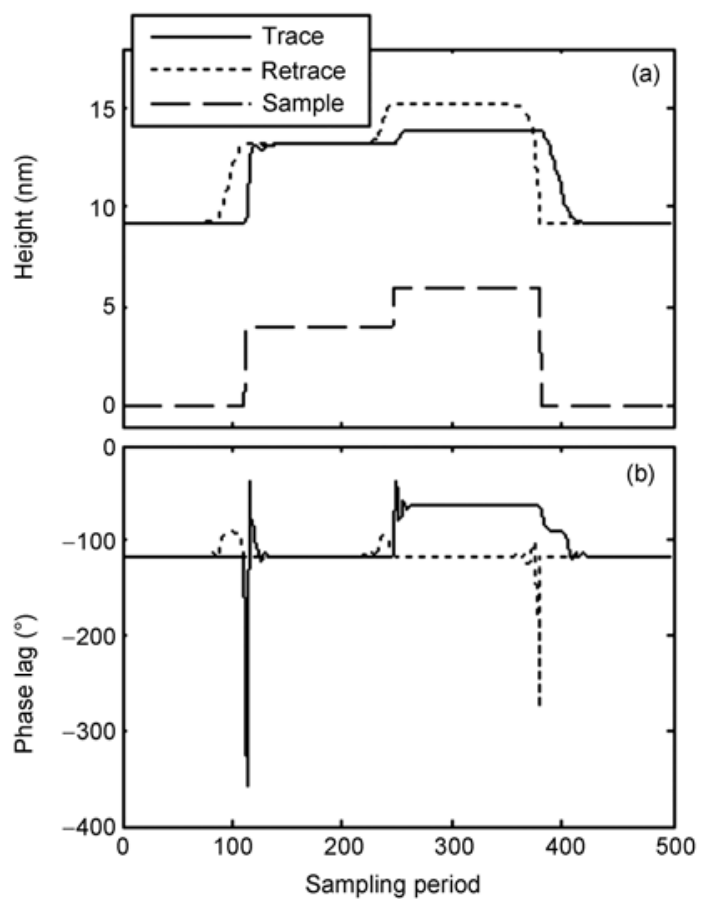

Figure 2 Bistability of the scanning curve. (a) Scanning image curve; (b) phase-shift curve. the tip scans the second step, the tip-sample distance decreases and the phase shift stabilizes at above $-90^{\circ}$. Therefore, the tip changes from low-state to high-state. However, in the backward curve, it is seen that the tip always works in the low state. As a result, there is deviation in the vertical height between the forward and backward curves of Figure 2(a). Therefore, using the phase-shift curve is an effective way to detect bistable behavior.

\subsection{Effect of excitation amplitude changes on tip motion}

If the phase difference between the tip motion signal and excitation signal is always bounded within $-90^{\circ}$ to $0^{\circ}$ and the tip always works in the high state, there is no state shift and imaging bistability is prevented. This can be achieved by adjusting the free amplitude. However, changing free amplitude affects the dependence curve of the amplitude and the position of equilibrium. Therefore, how to adjust the free amplitude to gain the best scanning image is discussed next. Here we set $A_{\text {set }}$ as $9 \mathrm{~nm}$.

The dependence of the tip-sample distance on the free amplitude under the same amplitude is shown in Figure 3(a). The range of free amplitude is $A_{\text {set }}-A_{\text {set }} \times 1.5$; i.e. $9-13.5 \mathrm{~nm}$.

The open circles in Figure 3(a) represent the curve of increasing free amplitude. When the free amplitude $A_{0}$ is less than or equal to $11.2 \mathrm{~nm}$, the tip works in the low state. When $A_{0}$ is greater than $11.2 \mathrm{~nm}$, the tip works in the high state.
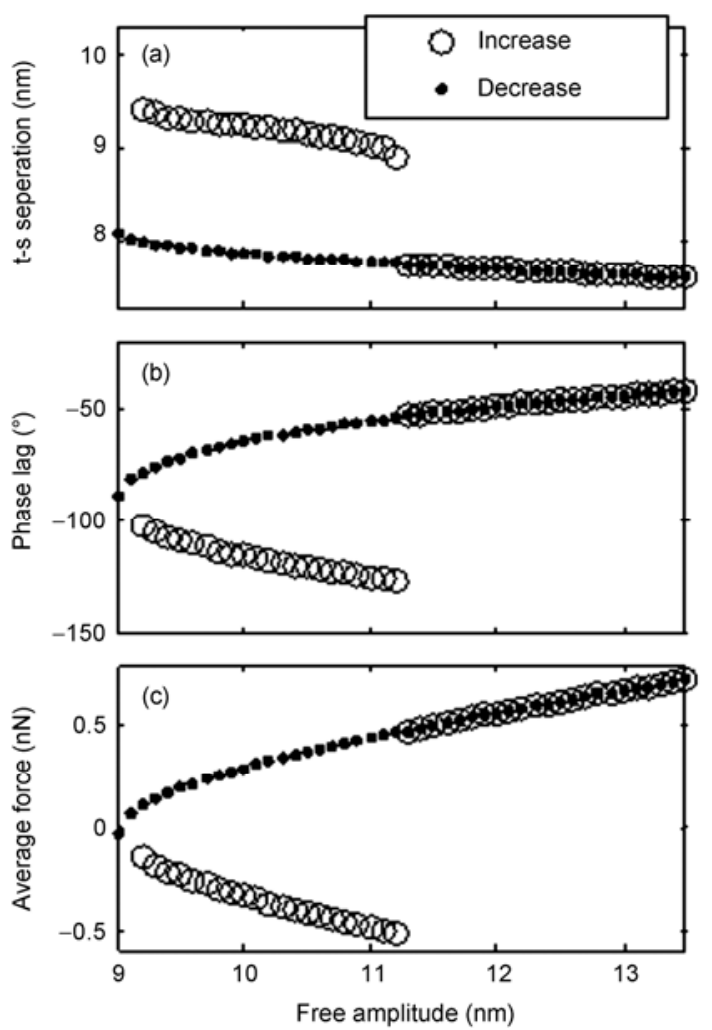

Figure 3 (a) Tip-sample separation; (b) amplitude; (c) average force dependence on free amplitude for the same set amplitude. 
Solid circles in Figure 3(a) represent the decreasing free amplitude, with the tip working in the high state. When the free amplitude $A_{0} \leqslant 9 \mathrm{~nm}$, the tip is in free oscillation and the curve may jump greatly at $9 \mathrm{~nm}$. Therefore, the minimum free amplitude should be slightly higher than $A_{\text {set }}$. In this work, the minimum free amplitude is $A_{\text {set }} \times 1.001$. The maximum tip-sample distance is $8.11 \mathrm{~nm}\left(A_{0}=9 \mathrm{~nm}\right)$ and the minimum tip-sample distance is $7.62 \mathrm{~nm}\left(A_{0}=13.5 \mathrm{~nm}\right)$. Therefore, the range is about $0.49 \mathrm{~nm}$. The new error $(0.49 \mathrm{~nm})$ is only $26.5 \%$ of the error $(1.85 \mathrm{~nm})$ caused by bistability.

The solid circles in Figure 3(b) show that the phase shift is always greater than $-90^{\circ}$. Therefore, the tip works in the high state and the phase shift decreases as the free amplitude decreases. Figure 3(b) also shows the curve of the increasing free amplitude (open circles) and the phase shift is less than $-90^{\circ}$. Therefore, the tip works in the low state and the phase shift decreases as the free amplitude increases. When the free amplitude increases to $11.2 \mathrm{~nm}$, the tip jumps from the low state to the high state, and the phase shift becomes greater than $-90^{\circ}$. Therefore, when the phase shift is less than $-90^{\circ}$, the tip may work in the low state. However, the tip's state jumps back to the high state with increasing free amplitude. Thus, we should force the tip to stay in the high state to prevent bistability.

The relationship between the free amplitude and the average force of the tip-sample interaction during a cycle is represented in Figure 3(c). When the tip works in the high state (solid circles), the phase shift decreases as the free amplitude decreases, and the average tip-sample force also reduces. Therefore, the phase shift should be set between $-90^{\circ}$ and $0^{\circ}$ to keep the tip in the high state. To make the average tip-sample force as small as possible, the phase shift should be set as close as possible to $-90^{\circ}$. However, the phase shift cannot be exactly $-90^{\circ}$ to avoid instability.

\section{Simulation and results}

On the basis of the above discussion, we should keep the tip in the high state by changing the free amplitude to prevent bistability. However, the free amplitude cannot be increased too greatly to avoid damaging the samples. In the following, a simulation system is built and the results are analyzed.

The framework of the simulation system is shown in Figure 4. The external loop is the same as in the amplitudemodulation mode. We compare the tip's actual amplitude with the set amplitude every 10 oscillation cycles (i.e. one sampling period) to obtain the error that is conveyed into the PID controller. The piezoelectric ceramic height is adjusted accordingly. In the internal loop, we compare the actual phase shift with the set phase shift every one oscillation cycle to obtain the error that is conveyed into the PID controller. We thus obtain a new free amplitude.

The design of the PID that control the free amplitude are

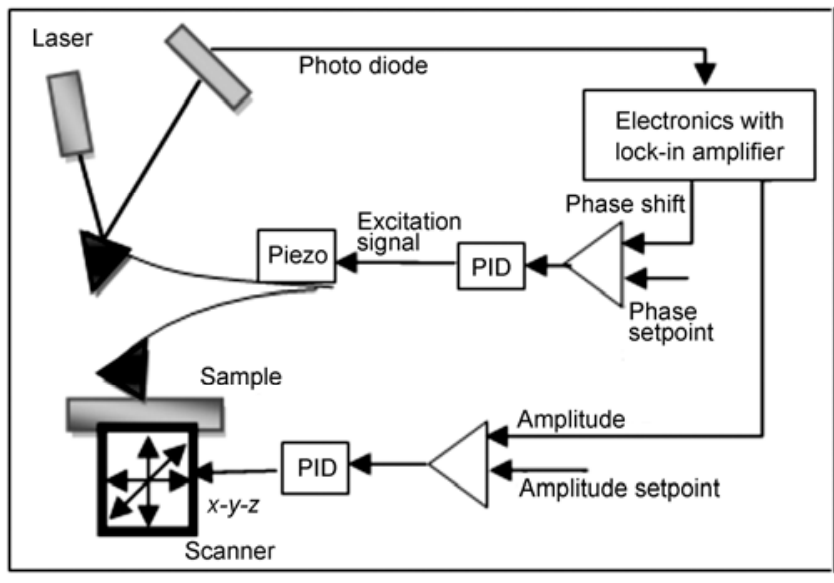

Figure 4 Schematic diagram of the simulation system.

as follows [27]:

$$
e(i)=\frac{\phi_{\text {setpoint }}-\phi(i)}{-\phi_{\text {setpoint }}} \text {. }
$$

Setting of the auxiliary value:

$$
\begin{aligned}
A_{\text {aux }}(i+1)= & A_{\text {aux }}(i)+k_{\mathrm{P}}[e(i)-e(i-1)] \\
& +k_{\mathrm{I}} e(i)+k_{\mathrm{D}}[e(i)-2 e(i-1)+e(i-2)] .
\end{aligned}
$$

New free amplitude:

$$
A_{0}(i)=A_{\text {set }} A_{\text {aux }}(i)
$$

The auxiliary value $A_{\text {aux }}$ is a parameter. Additionally, the original PID parameters are still available with the addition of the auxiliary value $A_{\text {aux }}$ even when the predetermined amplitude and quality factor change. To prevent system instability and contain the error caused by the change in free amplitude, the free amplitude is limited to between $A_{\text {set }} \times$ 1.001 and $A_{\text {set }} \times 1.5$.

On the basis of the above theory, we carry out a simulation using five coupled fourth-order Runge-Kutta algorithms. The chosen parameters are a set amplitude $A_{\text {set }}=9 \mathrm{~nm}$, phase shift $\varphi_{\text {setpoint }}=-70^{\circ}$, and free amplitude $A_{0} \approx 9.55 \mathrm{~nm}$ corresponding to Figure 3(b). The PID parameter values controlling the free amplitude are $k_{\mathrm{P}}^{*}=1, k_{\mathrm{I}}^{*}=0.002$, and $k_{\mathrm{D}}^{*}=2$, and the other parameters are the same as in Figure 2.

Figure 5 shows that the relationship between the amplitude and the tip-sample distance in the constant-phase-shift amplitude modulation is quite linear. Additionally, the two curves of increasing tip-sample distance and decreasing tip-sample distance overlap, indicating that the method can effectively eliminate bistability. With the tip's amplitude increasing, the free amplitude increases linearly, thus producing a constant phase shift.

The scanning curve of the simulation in the constantphase amplitude modulation is shown in Figure 6(a) and the 


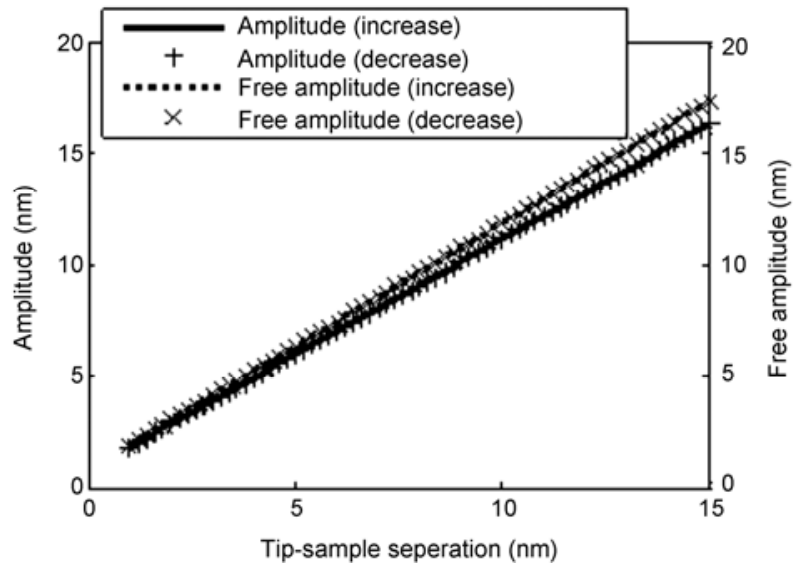

Figure 5 Amplitude curve for constant phase.

scanning curve compares better with Figure 2. The trace and retrace curves are at the same height. Figure 6(b) shows the phase-shift curve. The phase shift is mostly equal to $-70^{\circ}$, and the region of the phase shift being less than $-90^{\circ}$ is relatively narrow. Therefore, bistability has been effectively avoided.

In simulation, the ratio $\left(A_{\text {set }} / A_{0}\right)$ between the set amplitude and free amplitude is 0.9424 . This high ratio easily leads to bistability in traditional AM-AFM. Figure 3(c) shows that the higher the $A_{\text {set }} / A_{0}$ ratio, the smaller the interaction between tip and sample. However, when the phase shift is less than $-90^{\circ}$, as the tip scans the rising step of the sample, the free amplitude suddenly becomes large, making
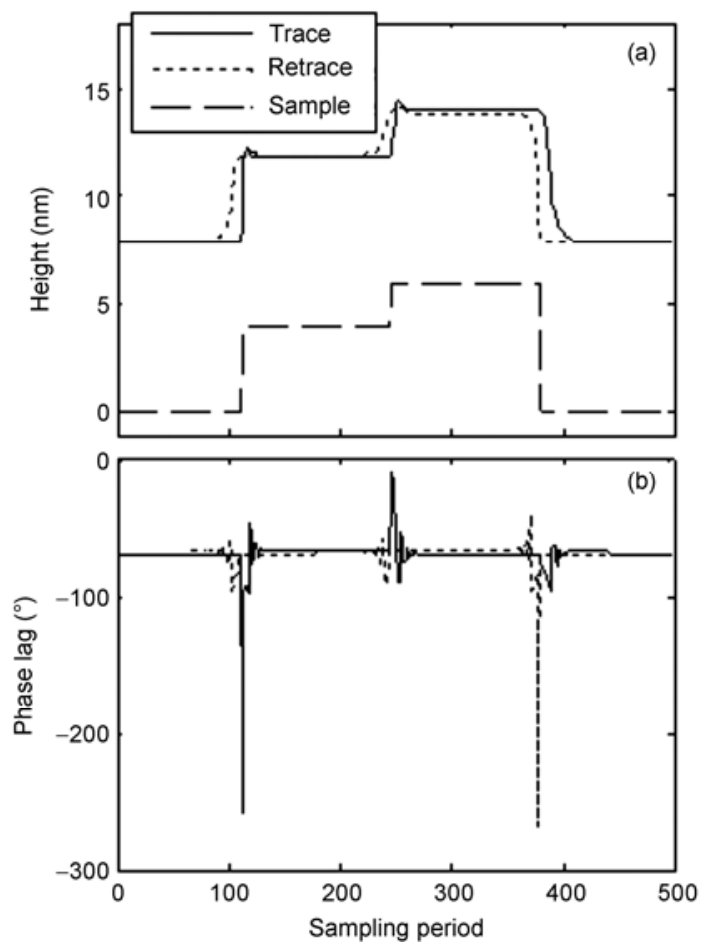

Figure 6 Simulation scanning curve in the constant-phase mode. the interaction force between the tip and sample slightly greater in the constant-phase mode than in conventional AM-AFM.

\section{Conclusion}

In this work, we propose AM-AFM with constant phase to prevent bistability. Simulation results show that the phase shift can distinguish high and low states of bistability. The high-state phase shift is within $-90^{\circ}$ to $0^{\circ}$, and the low-state phase shift is within $-180^{\circ}$ to $-90^{\circ}$. Therefore, when we adjust the free amplitude to limit the phase shift to a fixed value between $-90^{\circ}$ and $0^{\circ}$, we keep the tip in the high state, thereby preventing bistability.

Simulation analysis shows when the amplitude $A_{\text {set }}$ is set to $9 \mathrm{~nm}$, and the free amplitude changes between $A_{\text {set }}$ and $A_{\text {set }} \times 1.5$, the largest deviation in the balance position is only $26.5 \%$ of the scanning deviation caused by the bistability. Additionally, it is concluded that the tip can change indeed from a low to high state by increasing the free amplitude. The closer the set phase shift is to $-90^{\circ}$, the smaller the free amplitude and the smaller the tip-sample interaction. The simulation results verify the feasibility of the proposed system, and the overall performance is very good. The results indicate that AM-AFM in constant-phase mode is more stable than conventional AM-AFM.

This work was supported by the National Natural Science Foundation of China (11074019) and the National High-Tech Research and Development Program of China (2007AA12Z128).

1 Binnig G, Quate C, Gerber C. Atomic force microscopy. Phys Rev Lett, 1986, 56: 930-933

2 Yu A F, Qi Q, Jiang P, et al. Growth related carrier mobility enhancement of pentacene thin-film transistors with high-k oxide gate dielectric. Chin Phys Lett, 2009, 26: 078501

3 Han G Q, Zeng Y G, Yu J Z, et al. Evolution of Ge and SiGe quantum dots under excimer laser annealing. Chin Phys Lett, 2008, 25: 242-245

4 Yuan S, Dong Z L, Miao L, et al. Research on the reconstruction of fast and accurate AFM probe model. Chin Sci Bull, 2010, 55: 2750-2754

5 Wang C M, Sun J L, Long F, et al. Application of image alignment and time averaging methods in AFM detection for single DNA molecules. Chin Sci Bull, 2010, 55: 1613-1618

6 Martin Y, Williams C C, Wickramasinghe H K. Atomic force microscope-force mapping and profmng on a sub $100 \AA$ A scale. J Appl Phys, 1987, 61: 4723-4729

7 Marth M, Maier D, Honerkamp J, et al. A unifying view on some experimental effects in tapping-mode atomic force microscopy. $\mathrm{J}$ Appl Phys, 1999, 85: 7030-7036

8 García R, San Paulo A. Attractive and repulsive tip-sample interaction regimes in tapping-mode atomic force microscopy. Phys Rev B, 1999, 60: 4961-4967

9 García R, San Paulo A. Dynamics of a vibrating tip near or in intermittent contact with a surface. Phys Rev B, 2000, 61: R13381

10 Van der Water W, Molenaar J. Dynamics of vibrating atomic force microscopy. Nanotechnology, 2000, 11: 192-199 
11 Holscher H, Gotsmann B, Allers W, et al. Measurement of conservative and dissipative tip-sample interaction forces with a dynamic force microscope using the frequency modulation technique. Phys Rev B, 2001, 64: 075402

12 Holscher H, Gotsmann B, Allers W, et al. Comment on "Damping mechanism in dynamic force microscopy". Phys Rev Lett, 2002, 88: 019601

13 San Paulo A, García R. Unifying theory of tapping-mode atomicforce microscopy. Phys Rev B, 2002, 66: 041406

14 García R, Pérez R. Dynamic atomic force microscope methods. Surf Sci Rep, 2002, 47: 197-301

15 Solares S D. Eliminating bistability and reducing sample damage through frequency and amplitude modulation in tapping-mode atomic force microscopy. Meas Sci Tech, 2007, 18: 592-600

16 Solares S D. Frequency and force modulation atomic force microscopy: Low-impact tapping-mode imaging without bistability. Meas Sci Tech, 2007, 18: L9-L14

17 Li Y, Qian J Q. Higher harmonics generation in tapping mode atomic force microscope. Chin Phys Lett, 2009, 26: 100703

18 Li Y, Qian J Q, Li Y Z. Theory of higher harmonics imaging in tapping-mode atomic force microscopy. Chin Phys B, 2010, 19: 050701

19 Tamayo J, García R. Deformation, contact time, and phase contrast in tapping mode scanning force microscopy. Langmuir, 1996, 12: 4430-4435

20 Derjauin B V, Müller V M, Toporov Y P. Effect of contact deformations on the adhesion of particles. J Colloid Interface Sci, 1975, 53: 314-326

21 Israelachvili J. With Applications to Colloidal and Biological Systems Intermolecular and Surface Forces. New York: Academic, 1985

22 Cappella B, Dietler G. Force-distance curves by atomic force microscopy. Surf Sci Rep, 1999, 34: 1-104

23 Kühle A, Soerensen A H, Bohr J. Role of attractive forces in tapping tip force microscopy. J Appl Phys, 1997, 81: 6562-6569

24 Chen X, Davies M C, Roberts C J, et al. Interpretation of tapping mode atomic force microscopy data using amplitude-phase-distance measurements. Ultramicroscopy, 1998, 75: 171-181

25 Haugstad G, Jones R. Mechanisms of dynamic force microscopy on polyvinyl alcohol: Region-specific non-contact and intermittent contact regimes. Ultramicroscopy, 1999, 76: 77-86

26 García R, San Paulo A. Amplitude curves and operating regimes in dynamic atomic force microscopy. Ultramicroscopy, 2000, 82: 79-83

27 Pishkenari H N, Meghdari A. Surface defects characterization with frequency and force modulation atomic force microscopy using molecular dynamics simulations. Curr Appl Phys, 2010, 10: 583-591

Open Access This article is distributed under the terms of the Creative Commons Attribution License which permits any use, distribution, and reproduction in any medium, provided the original author(s) and source are credited. 